\title{
Comparative Population Genetics in the Human Gut Microbiome
}

7 Abstract

The genetic variation in the human gut microbiome is responsible for conferring a

9 number of crucial phenotypes like the ability to digest food and metabolize drugs. Yet, our

10 understanding of how this variation arises and is maintained remains relatively poor. Thus, the

11 microbiome remains a largely untapped resource, as the large number of co-existing species in

12 this microbiome presents a unique opportunity to compare and contrast evolutionary processes

13 across species to identify universal trends and deviations. Here we outline features of the human

14 gut microbiome that, while not unique in isolation, as an assemblage make it a system with

15 unparalleled potential for comparative population genomics studies. We consciously take a broad

16 view of comparative population genetics, emphasizing how sampling a large number of species

17 allows researchers to identify universal evolutionary dynamics in addition to new genes, which

18 can then be leveraged to identify exceptional species that deviate from general patterns. To

19 highlight the potential power of comparative population genetics in the microbiome, we re-

20 analyzed patterns of purifying selection across $\sim 40$ prevalent species in the human gut

21 microbiome to identify intriguing trends which highlight functional categories in the microbiome

22 that may be under more or less constraint. 


\section{Introduction}

The human microbiome is a complex ecosystem composed of hundreds of interacting species. Although the diversity of the microbiome has been extensively studied at a species level,

27 each species harbors genetic diversity that is quite varied across hosts as well as within a host

28 over time (Zhu et al. 2010, 2019; Schloissnig et al. 2013; Faith et al. 2013). This genetic

29 diversity can confer a number of crucial traits to microbes as well as their hosts, such as the

30 ability to digest food, metabolize drugs, and evade antibiotics. However, our understanding of

31 how these genetic variants arise and segregate via population genetic forces - e.g., random

32 genetic drift, mutation, recombination, selection, and migration - across the hundreds of species

33 that call our guts home, is relatively nascent (Garud \& Pollard 2020).

34 Our knowledge of how evolution proceeds in a community context is similarly

35 underdeveloped. Much of our intuition about the evolution of microorganisms come from

36 studying individual species (Good et al. 2017; Bruger \& Marx 2018; Herron \& Doebeli 2013;

37 Tenaillon et al. 2016; Xue et al. 2017; Lieberman et al. 2014). By contrast, the microbiome is

38 composed of hundreds of interacting species and strains in which both ecological and

39 evolutionary forces simultaneously act (Garud \& Pollard 2020; Good \& Hallatschek 2018).

40 Specifically, change in the frequency of an existing haplotype as well as the emergence of a new

41 haplotypes (i.e., evolution) can occur on the same timescale as changes in strain frequencies (i.e.,

42 ecology). These simultaneous ecological and evolutionary processes within the human gut

43 microbiome affords a unique opportunity. Rather than studying one species at a time, we can

44 study the population genetics and ecology of many ( $\gtrsim 40)$ species almost simultaneously. Thus,

45 the human gut microbiome is a model system for studying comparative population genetics

46 across co-existing species in a natural environment. 
Comparative population genetics is a rich area of study that has yielded insights into novel functional elements and population genetic processes in macroscopic organisms ranging al. 2010) to Drosophila melanogaster (Clark et al. 2007; Lawrie \& Petrov 2014). Now with the

51 availability of hundreds of thousands of new genomes from the deep sequencing of commensal

52 microbes (Pasolli et al. 2019; Almeida et al. 2019; Nayfach et al. 2019), similar comparative

53 analyses can be made across microbial species and populations. However, unlike typical

54 macroorganisms, the microbiome provides a rich opportunity to understand how ecological

55 interactions between species modulate evolutionary dynamics within individual species.

A model system for comparative population genetics: the human gut microbiome

The human gut microbiome is a compelling system for comparative population genetics.

59 The short time scales on which evolutionary dynamics occur in the microbiome make it possible to witness evolution in action as well as how evolution interacts with ecology (Zhao et al. 2019;

61 Garud et al. 2019; Yaffe \& Relman 2020; Poyet et al. 2019; Roodgar et al. 2020). Moreover, the

62 meta-population structure of human microbiomes lends itself naturally to treating measurements

63 in spatially distant hosts as independent biological replicates, from which we can decode general

64 principles. Given the massive amounts of data now available from thousands of individuals from

65 around the world (Almeida et al. 2019; Pasolli et al. 2019), the time is ripe for the study of

66 evolution in the microbiome. Additionally, the ability to experimentally manipulate this system

67 (Roodgar et al. 2020) as well as its members (Barroso-Batista et al. 2014; Zhao et al. 2019;

68 Ramiro et al. 2020) enables validation of computational predictions and discovery of new

69 principles. Finally, the strong relevance of the microbiome to our health makes it a medically 
70 important system (Thomas et al. 2019; Ley et al. 2005; Jakobsson et al. 2010; Duvallet et al.

71 2017). Thus, comparative population genetic studies in the microbiome will not only elucidate

72 general principles relevant to the microbiome, but also the broader field of population genetics.

73 Here we elaborate on these fundamental facets of the microbiome that make it the ideal system to

74 study comparative population genetics in a naturally complex ecosystem.

75

\section{1) Rapid evolution on short timescales}

With relatively short generation times of only( $\sim 1-10$ days in the human gut (Korem et al. 2015), microbes have the potential to rapidly evolve. This enables temporally resolved analyses that are almost impossible to replicate in charismatic macroscopic organisms. A sense of the evolutionary timescale of the human microbiome is best illustrated through a back-of-theenvelope calculation: taking the low end of the range of generation times for the gut microbiome of $\sim 1$ day (Korem et al. 2015; Milo \& Phillips 2016) we find that it has evolved for $\sim 9,000$ generations before its host has reached age 25 , a number that is on the order of the $\sim 10,000$ generations that humans have existed (Moorjani et al. 2016; Scerri et al. 2018). Alternatively stated, the microbiome within a human host can evolve on a timescale similar to that of the entire human species. The ability to sample populations over a large number of generations can alter how evolutionary biologists approach their questions. Instead of relying predominantly on phylogenetic reconstruction from static data, researchers can effectively observe evolution in real time. Over the course of just a few months, new genotypes can emerge and recombine over short time scales, and ultimately become lost or fixed over extended time scales. Indeed, it recently has 
93 been observed that adaptation and recombination can occur in the human gut over a matter of

94 months (Zheng et al. 2020; Yaffe \& Relman 2020; Poyet et al. 2019; Garud et al. 2019; Zhao et

95 al. 2019; Lin \& Kussell 2017). However, the necessary temporal resolution of sampling remains

96 subject to the researcher's question, as short-term adaptation in response to a temporary

97 environmental perturbation (e.g., a host consuming antibiotics over a few weeks) may require

98 denser sampling than evolution in a relatively unchanging environment.

\section{2) Replication across hosts}

Given that the human gut microbiome is a quasi-closed system, separate hosts can be

102 treated as replicate evolution studies, an observation that has been summarized by the captivating

103 moniker "seven billion microcosms" (Lieberman 2018). This level of replication can be

104 leveraged to identify targets of positive selection that recurrently accumulate fixation events in

105 independent hosts (i.e., parallel evolution; Xue et al. 2017; Bertels et al. 2019; Lieberman et al.

106 2014; Zhao et al. 2019; Poyet et al. 2019). By combining large cohort sizes with temporally

107 resolved sampling we can also examine how these signatures of parallelism change over time,

108 allowing us to dissect the temporal dynamics of adaptation (Barroso-Batista et al. 2014). For

109 example, targets of rapid adaptation typically harbor a disproportionate number of sites with

110 strong beneficial fitness effects, leading to the fixation of multiple mutations within a short

111 period of time (i.e., "coupon collecting"; Good et al. 2017). Alternatively, if the fitness effects of

112 sites in a gene depend on whether prior mutations have fixed elsewhere in the genome, then the

113 time between fixation events will be large (i.e., historical contingency; Gould 1990; Blount et al.

114 2008). The benefits of large cohorts are not limited to detecting adaptation. With large cohorts,

115 we can also observe deleterious alleles segregating at extremely low frequencies that are likely 
116 subject to purifyin selection (Lawrie \& Petrov 2014). Combined with exciting recent theoretical

117 developments (Neher \& Shraiman 2012; Nicolaisen \& Desai 2012; Cvijović et al. 2018; Good

118 2020), the human gut microbiome is an ideal system to examine the evolutionary dynamics of

119 purifying selection.

\section{3) Ecology and evolution frequently interact}

123 or become extinct, instead remaining at intermediate frequencies for extended periods of time

124 (Good et al. 2017; Good \& Hallatschek 2018). This "strain" level structure constitutes a form of

125 ecology that exists below the taxonomic level of species, and is commonly found within hosts for

126 most gut microbiota (Garud et al. 2019). The sheer prevalence of strain structure in the human

127 microbiome and the fact that they can differ on the order of a few nucleotides (Goyal et al. 2021)

128 suggests that ecological and evolutionary dynamics occur on similar timescales in microbial

129 systems, contrary to the historical belief that evolutionary timescales are longer than ecological

130 timescales (Slobodkin 1980). For example, strain frequencies can fluctuate on the same time

131 scale on which they acquire new genetic adaptations (Garud et al. 2019; Zhao et al. 2019). This

132 observation, along with the relative ease with which a large number of species can be sampled

133 across hosts, suggests that the human gut microbiome is a system with unmatched potential for

134 the exploration of eco-evolutionary dynamics.

135 The presence of overlapping ecological and evolutionary timescales in the microbiome

136 has spurred empirical and theoretical efforts to characterize eco-evolutionary interactions within

137 the gut. A prime example being a recent mathematical model that describes how the frequency of

138 a strain can change over time as de novo mutations accumulate, which affect how said strain 
consumes environmentally supplied resources in addition to its overall fitness (Good et al. 2018).

140 Thus, evolution can affect competition between strains with resource consumption as a

141 mediating factor, changes that ultimately alter community composition and structure. However,

142 it is unlikely that eco-evolutionary interactions within the gut can be sufficiently captured by

143 accounting for environmentally supplied resources alone. Rather, microorganisms often secrete

144 secondary metabolic compounds, supplying additional resources that can be consumed by other

145 species (i.e., cross-feeding). This metabolic dependency promotes species co-existence and

146 becomes increasingly likely in communities with many species, an ecological bedrock that

147 supports subsequent coevolution (D'Souza et al. 2018; Lilja \& Johnson 2019). The widespread

148 nature of this phenomenon may explain empirical patterns where it appears as though the arrow

149 of causation between evolution and ecology is reversed, a prominent example being that the

150 diversification rate of a species is correlated with the number of species in its community (Madi

151 et al. 2020).

152

\section{4) Experimental manipulation}

While a natural system enables researchers to study complex phenomena that cannot be

recapitulated exactly in the laboratory, some degree of experimental manipulation is necessary to

156 validate predictions and generate new insights. Over the last few years, substantial progress has

157 been made towards characterizing the evolutionary dynamics of adaptation in microbial

158 populations. The use of lineage tracing via barcoding has allowed the distribution of fitness

159 effects of de novo mutations to be quantified in certain species (Levy et al. 2015) in addition to

160 providing evidence that the travelling wave is an appropriate model of microbial adaptation

161 (Nguyen Ba et al. 2019). The ease with which such techniques can be applied to non-model 
162 species varies, though gene deletion via transposon mutagenesis libraries has been shown to be

163 particularly effective for identifying loci that confer a growth advantage in environments with

164 different resources (Cain et al. 2020), different sets of co-occurring species (Thibault et al. 2019),

165 for species isolated from the gut (Ruiz et al. 2013), and in the gut microbiome of model

166 organisms (Powell et al. 2016; Zimmermann et al. 2019; Ludington \& Ja 2020; Barreto et al.

167 2020; Barroso-Batista et al. 2020). These experiments can serve as a compliment to traditional

168 comparative population genetic analyses, allowing us to test hypotheses formed from

169 metagenomic observational studies. While all these studies fall short of true in vivo manipulation

170 of human guts, they are useful approximations that allow for high-throughput manipulations to

171 be performed and evolutionary and ecological hypotheses to be tested.

172

5) Relevance to health

A significant benefit to studying comparative population genetics in the human gut

175 microbiome is that the findings made may have a direct relevance for human health. The species

176 composition of the gut microbiome is known to be essential for proper immunological (Belkaid

177 \& Hand 2014), neurological (Yano et al. 2015), and metabolic development (Rowland et al.

178 2018), and is associated with a number of human diseases including colorectal cancer, diabetes

179 (Vallianou et al. 2018), and obesity (Ley et al. 2005, 2006). While the connection of the human

180 microbiome to host health has been primarily studied at the species level, genetic variants in the

181 microbiome play a crucial role for health as well. Specifically, microbiome genetic variants can 182 confer a number of critical traits to human hosts, including the digestion of new foods (Kenny et

183 al. 2020; Hehemann et al. 2010), antibiotic resistance (Gautam et al. 2018), and the

184 metabolization of drugs (Spanogiannopoulos et al. 2016). A comparative genomics approach will 
enable the discovery of new microbiome genetic variants (Sberro et al. 2019), which may

ultimately be useful for the future development of effective microbiome therapies.

\section{Lessons from comparative population genetics in the microbiome}

While our understanding of the microbiome and the discipline of comparative population

genetics have rapidly expanded since the emergence of next-generation sequencing almost two

population genetics in the microbiome is still being realized. Here we present three goals for the microbiome, and 3) individual species and genomic features that deviate from general patterns.

\section{1) Inference of Functionality}

Currently, the annotation of genes in the microbiome and our understanding of their

functionality is severely lacking, with an estimated $40 \%$ being "hypothetical" (Almeida et al.

200 2019). Comparative population genetics has the potential to shed light on the functions of

201 existing hypothetical genes and assist with the identification of new ones. Much of the utility of

202 comparative population genetics derives from the neutral theory of molecular evolution, which

203 predicts that if mutations in functional regions of genomes tend to be deleterious, those regions

204 will evolve at a slower rate than effectively neutral nonfunctional regions (Kimura 1983). This

205 constraint allows for conserved elements of the genome to be identified between highly diverged

206 species; a "phylogenetic footprint” (Lawrie \& Petrov 2014). Using this basic assumption,

207 comparative genomic analyses across groups of macroorganisms as diverse as Drosophila and 
mammals have yielded insight into novel proteins (Clark et al. 2007; Lawrie \& Petrov 2014).

209 Now, recent efforts have been made to apply this approach to the microbiome (Fremin \& Bhatt

210 2020). Specifically, Sberro et al. (2019) recently performed a comparative analysis on shotgun

211 microbiome metagenomic data and discovered thousands of novel small genes. Among their

212 discoveries was a novel small ribosome-associated protein that seems to be transcribed and

213 translated at high levels. Despite the fundamental functional significance of this protein, it may

214 have been missed due to the historical focus on model organisms such as E. coli and common

215 pathogens.

216 However, there is additional justification to claim that microorganisms harbor a

217 substantial number of unannotated functional elements. Population genetic theory coupled with

218 cellular energetics predicts that the vast majority of unannotated genes within the gut

219 microbiome likely play some functional role (Lynch \& Marinov 2015; Martinez-Gutierrez \&

220 Aylward 2019). For example, a single nonfunctional nucleotide within a microbial genome is

221 visible to purifying selection (Lynch \& Marinov 2015), a stark contrast to macroorganisms

222 where junk DNA is highly prevalent (Lynch 2007). Coupled with the higher gene density in

223 microorganisms due to overlapping open reading frames (Johnson \& Chisholm 2004), this

224 prediction suggests that the gut microbiome is a particularly apt system for researchers who wish

225 to leverage statistical evidence provided by comparative population genomics to confirm the

226 purported functionality of a given gene. Indeed, researchers are likely already acting on this

227 prediction, as recent efforts combined comparative genomics with RNA-seq to identify 2,000

228 novel structural RNAs in the microbiome (Fremin \& Bhatt 2020). With hundreds of species

229 harboring genomes with high gene density across billions of hosts, the gut microbiome is still 
very much a proverbial "gold rush" for the discovery and characterization of novel proteins and

231

232

233

234

235

236

237

238

239

240

241

242

243

244

245

246

247

248

249

250

251

252

RNAs.

\section{2) Robust evolutionary patterns}

Arguably, it is necessary to gain some degree of knowledge regarding the typical evolutionary dynamics of a species in a given system before comparisons between species can be performed. At first glance, it would appear as if the goal of identifying general evolutionary patterns in the human gut microbiome is hopeless. There are few cases where population geneticists would say that we have sufficient knowledge of the evolutionary dynamics of one species, much less hundreds or thousands of species that interact in the same environment. Operating under this assumption, we would conclude that the complexity of the microbiome is irreducible. This is not entirely an unjustified claim, since if one is interested in the evolutionary dynamics of an individual species, how can those dynamics be sufficiently characterized if you cannot examine an isolated species in vivo?

The fault here is the idea that we need to understand the dynamics of individual species to understand the general dynamics of the system. Instead, progress can be made by temporarily abandoning the Cartesian framework that is ubiquitous in traditional biology (Levins \& Lewontin 1987) and embracing an alternative approach, where we exchange determinism for a statistical property, the average over an ensemble of species. This rationale is essentially what physicists realized in the $19^{\text {th }}$ century (Pathria $\&$ Beale 2011) and has been applied in recent years to examine the ecological dynamics of microorganisms, through the development of mathematical models (Advani et al. 2018; Barbier \& Arnoldi 2017) as well as the investigation of empirical data (Ji et al. 2020; Grilli 2020; Descheemaeker \& de Buyl 2020). It stands to 
253 reason that comparative population genetics could learn from such an approach. While our

254 argument here is primarily statistical, a spiritually similar argument has been made regarding the

255 use of effective models that coarse-grain over taxonomic details to identify quantitative patterns

256 in microbial ecology and evolution (Good \& Hallatschek 2018).

257 Ultimately it is necessary to take stock of the set of patterns that remain robust across

258 phylogenetically diverged species within the human gut, allowing us to identify the evolutionary

259 dynamics that universally occur. Here, we will briefly examine a few notable evolutionary

260 patterns that have been observed across species.

261

\section{i. Population Structure}

The genetic composition of commensal bacteria varies considerably from host to host

264 (Schloissnig et al. 2013; Truong et al. 2017; Costea et al. 2017), suggesting that bacteria do not

265 rampantly migrate between hosts. Instead, for each species, hosts are typically colonized by a

266 handful of strains that seem to be unique to each host (Garud et al. 2019; Schloissnig et al. 2013).

267 The typical number of strains within a species is variable, likely reflecting the degree that strains

268 can diverge and evolve sufficient ecological differences necessary to co-exist (Good et al. 2018).

269 Though this within-host population structure does not seem to necessarily have bearing on

270 across-host population structure, as the global biogeography of genetic variants can vary

271 considerably across species. For example, the genetic diversity of Eubacterium rectale mirrors

272 the genetic diversity of hosts (Truong et al. 2017; Tett et al. 2019; Nayfach et al. 2016; Costea et

273 al. 2017; Karcher et al. 2020), whereas species such as B. vulgatus seem to show little or no

274 geographic structure. The mechanisms responsible for variation in the global biogeography of

275 species remains unclear. Vertical transmission from parents to infants may contribute, as strains 
276 from certain species are more likely to colonize and persist in infant guts, the genera Bacteroides

277 and Bifidobacterium being noted examples (Lou et al. 2021). Though the benefit of being the

278 first to colonize a host is likely temporary, as the majority of strains are replaced over several

279 decades (Garud et al. 2019). Alternative mechanisms for varied levels of biogeography include

280 traits that promote airborne transmission being restricted to certain lineages (Brown 2000)

281 which may explain the variation in transmission rates among species (Brito et al. 2019), the

282 interaction of the microbiome with the genetics of its host (Goodrich et al. 2016), and even the

283 presence of spatial structure itself, which may promote the preservation of genetic variation

284 (Pearce \& Fisher 2019), though these hypotheses remain to be fully tested.

\section{ii. Recombination}

Although all bacteria reproduce clonally, the degree to which bacteria recombine varies

widely. The recombination rate of a species determines whether populations evolve primarily via

290 that are effectively independent (Neher \& Shraiman 2011), which can have consequences for

291 whether gene-specific versus genome-wide selective sweeps are more common (Shapiro et al.

292 2012; B. Jesse Shapiro 2016; Bendall et al. 2016). To quantify recombination in

293 bacteria, researchers have begun to characterize the statistical association of alleles at different

294 loci (i.e., linkage disequilibria), where the degree of association can be viewed a function of the

295 recombination rate. For several species found in the gut, as well as environmental samples and

296 pathogens, linkage disequilibria tends to decay as the genetic distance between a pair of loci

297 increases, which suggests that recombination may be common (Crits-Christoph et al. 2020;

298 Sakoparnig et al. 2021; Lin \& Kussell 2019). Such rampant recombination suggests that while 
microbes reproduce clonally, the label "asexual" is a misnomer. Instead, microorganisms are increasingly being deemed as "quasi-sexual", where a large number of loci evolve independently instead of as genotypes despite the clonal nature through which they are reproduced (Smith et al. 2009), while others are likely subject to additional evolutionary forces that can generate correlations between sites, such as demographic history and selection. Selection seems to play a

307 particularly prominent role, where recent developments in population genetic theory provide the groundwork necessary for subsequent empirical investigation (Arnold et al. 2020; Good 2020).

309 These forces will need to be disentangled to understand the full extent of recombination in the 310 microbiome.

iii. Short term evolution within hosts

Recently, it was found that evolutionary changes can occur in the human gut microbiome

314 on short timescales of just a few months and even days(Garud et al. 2019; Ghalayini et al. 2018;

315 Roodgar et al. 2020; Zhao et al. 2019; Poyet et al. 2019; Yaffe \& Relman 2020), and that strain

316 replacements are generally rare over that timescale. These evolutionary changes modify the

317 haplotypes of existing lineages and seem to derive from a mixture of de novo mutations and

318 horizontal gene transfer via recombination. The recombination-seeded events are a unique mode 319 of adaptation that highlight how a complex community can maintain a reservoir of adaptive 320 genetic material, which may be particularly useful in rapidly fluctuating environments where

321 evolution via de novo mutations may take a long time. Thus, complex communities may be able 
322 to modulate the mode and tempo of evolution of focal species (Madi et al. 2020). At longer time-

323 scales, these evolutionary changes tend to give way to ecology, as strain replacements become

324 common. So far, there does not seem to be any evidence that rates of evolution or strain

325 replacement differ across species, though future work may identify any differences. Though

326 these seemingly contrasting dynamics raise the broader point that being able to examine

327 evolutionary dynamics across a range of timescales will ultimately require researchers to intuit

328 what evolutionary and ecological dynamics are relevant on a given timescale and, ultimately,

329 construct models that bridge separate dynamics.

\section{iv. $\quad$ Purifying selection}

By comparing haplotypes of a given species from different hosts, we can focus on

333 patterns that are the outcome of evolutionary dynamics that have operated over an extended

334 timescale. One such pattern is how the ratio of nonsynonymous to synonymous divergences

$335\left(d_{N} / d_{S}\right)$ changes as synonymous divergence $\left(d_{S}\right)$ increases, which would indicate in what

336 direction selection tends to dominate over an extended timescale. Looking at empirical data from

337 the microbiome, it is clear that $d_{N} / d_{S}$ tends to decrease with increasing $d_{S}$, suggesting that

338 purifying selection tends to dominate as lineages diverge (Fig. 1a; Garud et al. 2019).

339 Surprisingly, the shape of the relationship can be captured by an effective model of selection

340 composed of two parameters: a single selection coefficient and the fraction of sites subject to

341 selection (Garud et al. 2019). Some species clearly have values of $d_{N} / d_{S}$ that are further from

342 this prediction than others, an observation that we will return to below. But as a first

343 approximation we can say that purifying selection explains genome-wide patterns of genetic

344 divergence across species within the gut (Fig. 1a). 


\section{3) Identifying deviations from general trends}

It may not be clear how a researcher can leverage universal evolutionary patterns to

348 identify genes and species of interest. Indeed, interest in identifying exceptional species and

349 targets of evolution is likely why many researchers compare species in the first place (Lawrie \&

350 Petrov 2014; Leffler et al. 2012; Huber et al. 2020). Here, we will re-examine the relationship

351 between $d_{S}$ and $d_{N} / d_{S}$ discussed earlier to illustrate how starting with a strong universal pattern

352 can provide a backdrop against which we identify deviations from the overall trend. We see that

353 certain species tend to fall above or below the prediction of an effective model of purifying

354 selection (Fig. 1a). To identify species that are subject to stronger or weaker purifying selection

355 than expected by chance, we first coarse-grain genes by their annotated metabolic pathways,

356 providing a set of variables shared across species. We can then permute species-level

357 observations within a given pathway and establish 95\% confidence intervals, a non-parametric

358 test that allows us to identify species with exceptional $d_{N} / d_{S}$ (Fig. 1b).

359 Though we coarse-grained genes by necessity, it allowed us to perform additional

360 analyses in which we leverage information across multiple species. First, we can see that certain

361 pathways typically have lower $d_{N} / d_{S}$ than others, suggesting that they are subject to stronger

362 purifying selection (Fig. 1c). We can then identify pathways that are subject to stronger or

363 weaker purifying selection than expected by chance by permuting values of $d_{N} / d_{S}$ across

364 pathways within each species and establishing confidence intervals (see Supplemental

365 Information). The results of this test align with our biological intuition, as essential pathways

366 tend to be under stronger purifying selection (e.g., glycolysis, nucleotide biosynthesis, Krebs

367 cycle, etc.), while pathways that rely on specific resources (e.g., sulfur metabolism) tend to be 
under relatively relaxed selection, an observation consistent with prior analyses using polymorphism data (Schloissnig et al. 2013).

371 species, we can continue our comparative population genetic analyses and examine the statistical

372 properties of $d_{N} / d_{S}$ across pathways. First, we can determine whether the relative spread of

$373 d_{N} / d_{S}$ remains similar across pathways by examining whether the ratio of the standard deviation

374 to the mean (i.e., the coefficient of variation) remains constant. We find that this is the case, as

375 the mean $d_{N} / d_{S}$ of a pathway $\left(\left\langle d_{N} / d_{S}\right\rangle\right)$ across species is linear with respect to its variance

$376\left(\sigma_{d_{N} / d_{S}}^{2}\right.$; Fig. 1b). This observation is reminiscent of Taylor's Law (Taylor 1961), a pattern often

377 found in ecological systems (Grilli 2020). Similar to Taylor's Law, we find that the slope of this

378 relationship is not significantly different from two $(t=0.684 ; P=0.248)$, which we can

379 interpret as the mean being equal to the standard deviation across pathways $(\mathrm{CV}=$

$\left.380 \sigma_{d_{N} / d_{S}}^{2} /\left\langle d_{N} / d_{S}\right\rangle^{2}=1\right)$. This observation suggests that the relative dispersion of $d_{N} / d_{S}$ remains

381 constant as the overall level of constraint within a pathway is relaxed. Though values of $d_{N} / d_{S}$

382 across pathways are not independent, as the correlation in $d_{N} / d_{S}$ across pathways for a given

383 pair of species tends to decay with phylogenetic distance $\left(\beta=-0.104, P<10^{-6}\right)$, suggesting that

384 the strength of purifying selection within a given essential pathway is moderately conserved

385 through evolutionary time. However, this does not provide an explanation of why certain

386 pathways are subject to stronger purifying selection than others, or why the strength of selection

387 varies across lineages, a question that can likely only be answered by incorporating additional

388 biological details about the pathways and species themselves (Bielawski \& Yang 2004; Aguileta

389 et al. 2009). Rather, it illustrates how investigating deviations from an empirical pattern can lead

390 to novel findings. 


\section{Future directions for comparative population genetics in the microbiome}

The study of comparative population genetics in the human microbiome is nascent and

394 full of potential. There are multiple avenues of progress in the microbiome field that will benefit comparative population genetics as a discipline. First, advances in sequencing technology will

396 allow us to refine our estimates of important quantities. For example, long-read technologies,

397 such as nanopore metagenomic sequencing, will allow researchers to quantify linkage between

398 physically distant sites (Bharti \& Grimm 2019; Zlitni et al. 2020; Yaffe \& Relman 2020; Karst et

399 al. 2021), providing higher resolution to uncover fundamental evolutionary processes of

400 recombination, mutation, and adaptation within and across species. These advances, coupled

401 with decreasing costs of library preparation (Baym et al. 2015), will allow researchers to sample

402 large cohorts over time and observe how genotypes dissipate into alleles and reemerge via

403 recombination over their sojourn times, enabling us to build more detailed evolutionary models.

404 Second, the fact that the gut exists as a physical environment is often overlooked.

405 Environmental factors such as temperature (Groussin \& Gouy 2011) as well as spatial structure

406 (Tropini et al. 2017) can affect the evolutionary dynamics of microbial species. Even deceptively

407 simple features of the gut such as its resemblance to a chemostat or the peristaltic mixing that

408 arises due to digestion can produce complex ecological and evolutionary dynamics (Locey \&

409 Lennon 2019; Cremer et al. 2016).

Finally, there is arguably as much a need to examine the targets of molecular evolution as

412 the general processes shaping genetic variation in the microbiome. The genes that contribute to

413 adaptation ultimately encode physical aspects of cells, which means that subsequent experiments 
414 will be necessary to gain a more complete understanding on how adaptation in the gut proceeds

415 and varies across species (Lynch \& Trickovic 2020; Lynch et al. 2014). These advances and

416 considerations will allow researchers to understand the general evolutionary dynamics of the

417 microbiome, expanding the breadth and depth of comparative population genetics as a

418 discipline

419

420 Data Availability

421 The raw sequencing reads for the metagenomic samples used in this study were previously

422 described (Garud, et al., 2019). The source code for figure generation and associated metadata is

423 available on GitHub: https://github.com/garudlab/CompPopGenMicrobiomeReview.

424

425 Acknowledgments

426 We thank Kirk Lohmueller for helpful discussions and John Connoly and Leah Briscoe for

427 providing feedback on an earlier draft. This work was supported by the NSF Postdoctoral

428 Research Fellowships in Biology Program under Grant No. 2010885. 


\section{References}

430 Advani M, Bunin G, Mehta P. 2018. Statistical physics of community ecology: a cavity solution

431 to MacArthur's consumer resource model. J. Stat. Mech. 2018:033406. doi: 10.1088/1742-

$4325468 / \mathrm{aab} 04 \mathrm{e}$.

433 Aguileta G, Refrégier G, Yockteng R, Fournier E, Giraud T. 2009. Rapidly evolving genes in

434 pathogens: methods for detecting positive selection and examples among fungi, bacteria, viruses

435 and protists. Infect Genet Evol. 9:656-670. doi: 10.1016/j.meegid.2009.03.010.

436 Almeida A et al. 2019. A new genomic blueprint of the human gut microbiota. Nature. 568:499-

437 504. doi: 10.1038/s41586-019-0965-1.

438 Arnold B et al. 2020. Fine-Scale Haplotype Structure Reveals Strong Signatures of Positive

439 Selection in a Recombining Bacterial Pathogen. Mol Biol Evol. 37:417-428. doi:

$440 \quad 10.1093 / \mathrm{molbev} / \mathrm{msz} 225$.

441 Barbier M, Arnoldi J-F. 2017. The cavity method for community ecology. Ecology doi:

$442 \quad 10.1101 / 147728$.

443 Barreto HC, Sousa A, Gordo I. 2020. The Landscape of Adaptive Evolution of a Gut Commensal

444 Bacteria in Aging Mice. Current Biology. 30:1102-1109.e5. doi: 10.1016/j.cub.2020.01.037.

445 Barroso-Batista J et al. 2020. Specific Eco-evolutionary Contexts in the Mouse Gut Reveal

446 Escherichia coli Metabolic Versatility. Current Biology. 30:1049-1062.e7. doi:

$447 \quad 10.1016 /$ j.cub.2020.01.050. 
448 Barroso-Batista J et al. 2014. The First Steps of Adaptation of Escherichia coli to the Gut Are

449 Dominated by Soft Sweeps. PLoS Genetics. 10:e1004182. doi: 10.1371/journal.pgen.1004182.

450 Baym M et al. 2015. Inexpensive Multiplexed Library Preparation for Megabase-Sized Genomes

451 Green, SJ, editor. PLoS ONE. 10:e0128036. doi: 10.1371/journal.pone.0128036.

452 Belkaid Y, Hand TW. 2014. Role of the microbiota in immunity and inflammation. Cell.

453 157:121-141. doi: 10.1016/j.cell.2014.03.011.

454 Bendall ML et al. 2016. Genome-wide selective sweeps and gene-specific sweeps in natural

455 bacterial populations. ISME Journal. 10:1589-1601. doi: 10.1038/ismej.2015.241.

456 Bertels F, Leemann C, Metzner KJ, Regoes RR. 2019. Parallel Evolution of HIV-1 in a Long-

457 Term Experiment. Molecular Biology and Evolution. 36:2400-2414. doi:

$458 \quad 10.1093 / \mathrm{molbev} / \mathrm{msz} 155$.

459 Bharti R, Grimm DG. 2019. Current challenges and best-practice protocols for microbiome 460 analysis. Briefings in Bioinformatics. doi: 10.1093/bib/bbz155.

461 Bielawski JP, Yang Z. 2004. A Maximum Likelihood Method for Detecting Functional

462 Divergence at Individual Codon Sites, with Application to Gene Family Evolution. J Mol Evol.

463 59:121-132. doi: 10.1007/s00239-004-2597-8

464 Blount ZD, Borland CZ, Lenski RE. 2008. Historical contingency and the evolution of a key

465 innovation in an experimental population of Escherichia coli. PNAS. 105:7899-7906. doi:

$466 \quad 10.1073 /$ pnas.0803151105. 
467 Brito IL et al. 2019. Transmission of human-associated microbiota along family and social

468 networks. doi: 10.1038/s41564-019-0409-6.

469 Brown LM. 2000. Helicobacter pylori: Epidemiology and routes of transmission. Epidemiologic

470 Reviews. 22:283-297. doi: 10.1093/oxfordjournals.epirev.a018040.

471 Bruger EL, Marx CJ. 2018. A decade of genome sequencing has revolutionized studies of

472 experimental evolution. Current Opinion in Microbiology. 45:149-155. doi:

473 10.1016/j.mib.2018.03.002.

474 Cain AK et al. 2020. A decade of advances in transposon-insertion sequencing. Nature Reviews

475 Genetics. 21:526-540. doi: 10.1038/s41576-020-0244-X.

476 Clark AG et al. 2007. Evolution of genes and genomes on the Drosophila phylogeny. Nature.

477 450:203-218. doi: 10.1038/nature06341.

478 Costea PI et al. 2017. Subspecies in the global human gut microbiome. Molecular Systems

479 Biology. 13:960. doi: 10.15252/msb.20177589.

480 Cremer J et al. 2016. Effect of flow and peristaltic mixing on bacterial growth in a gut-like

481 channel. PNAS. 113:11414-11419. doi: 10.1073/pnas.1601306113.

482 Crits-Christoph A, Olm MR, Diamond S, Bouma-Gregson K, Banfield JF. 2020. Soil bacterial

483 populations are shaped by recombination and gene-specific selection across a grassland meadow.

484 The ISME Journal. 14:1834-1846. doi: 10.1038/s41396-020-0655-x.

485 Cvijović I, Good BH, Desai MM. 2018. The Effect of Strong Purifying Selection on Genetic 486 Diversity. Genetics. 209:1235-1278. doi: 10.1534/genetics.118.301058. 
487 Davydov EV et al. 2010. Identifying a High Fraction of the Human Genome to be under

488 Selective Constraint Using GERP++. PLOS Computational Biology. 6:e1001025. doi:

489 10.1371/journal.pcbi.1001025.

490 Descheemaeker L, de Buyl S. 2020. Stochastic logistic models reproduce experimental time

491 series of microbial communities Krishna, S \& Walczak, AM, editors. eLife. 9:e55650. doi:

$49210.7554 /$ eLife.55650.

493 D'Souza G et al. 2018. Ecology and evolution of metabolic cross-feeding interactions in bacteria.

494 Nat. Prod. Rep. 35:455-488. doi: 10.1039/C8NP00009C.

495 Duvallet C, Gibbons SM, Gurry T, Irizarry RA, Alm EJ. 2017. Meta-analysis of gut microbiome

496 studies identifies disease-specific and shared responses. Nature Communications. 8:1784. doi:

$497 \quad 10.1038 / \mathrm{s} 41467-017-01973-8$.

498 Faith JJ et al. 2013. The long-term stability of the human gut microbiota. Science. 341. doi:

$499 \quad 10.1126 /$ science. 1237439.

500 Fremin BJ, Bhatt AS. 2020. A combined RNA-Seq and comparative genomics approach

501 identifies 1,085 candidate structured RNAs expressed in human microbiomes. bioRxiv.

$502 \quad$ 2020.03.31.018887. doi: 10.1101/2020.03.31.018887.

503 Garud NR, Good BH, Hallatschek O, Pollard KS. 2019. Evolutionary dynamics of bacteria in the 504 gut microbiome within and across hosts Gordo, I, editor. PLoS Biol. 17:e3000102. doi:

505 10.1371/journal.pbio.3000102. 
506 Garud NR, Pollard KS. 2020. Population Genetics in the Human Microbiome. Trends in

507 Genetics. 36:53-67. doi: 10.1016/j.tig.2019.10.010.

508 Gautam A et al. 2018. Altered fecal microbiota composition in all male aggressor-exposed

509 rodent model simulating features of post-traumatic stress disorder. Journal of Neuroscience

510 Research. 96:1311-1323. doi: 10.1002/jnr.24229.

511 Ghalayini $\mathrm{M}$ et al. 2018. Evolution of a dominant natural isolate of Escherichia coli in the human

512 gut over the course of a year suggests a neutral evolution with reduced effective population size.

513 Applied and Environmental Microbiology. 84. doi: 10.1128/AEM.02377-17.

514 Good BH. 2020. Linkage disequilibrium between rare mutations. bioRxiv. 2020.12.10.420042.

515 doi: $10.1101 / 2020.12 .10 .420042$

516 Good BH, Hallatschek O. 2018. Effective models and the search for quantitative principles in

517 microbial evolution. Current Opinion in Microbiology. 45:203-212. doi:

$518 \quad$ 10.1016/j.mib.2018.11.005.

519 Good BH, Martis S, Hallatschek O. 2018. Adaptation limits ecological diversification and

520 promotes ecological tinkering during the competition for substitutable resources. Proc Natl Acad

521 Sci USA. 115:E10407-E10416. doi: 10.1073/pnas.1807530115.

522 Good BH, McDonald MJ, Barrick JE, Lenski RE, Desai MM. 2017. The dynamics of molecular

523 evolution over 60,000 generations. Nature. 551:45-50. doi: 10.1038/nature24287.

524 Goodrich JK, Davenport ER, Waters JL, Clark AG, Ley RE. 2016. Cross-species comparisons of 525 host genetic associations with the microbiome. doi: 10.1126/science.aad9379. 
526 Gould SJ. 1990. Wonderful life: the Burgess Shale and the nature of history. Norton \& Co: New

527 York.

528 Goyal A, Bittleston LS, Leventhal GE, Lu L, Cordero OX. 2021. Interactions between strains

529 govern the eco-evolutionary dynamics of microbial communities. bioRxiv. 2021.01.04.425224.

530 doi: $10.1101 / 2021.01 .04 .425224$.

531 Grilli J. 2020. Macroecological laws describe variation and diversity in microbial communities.

532 Nature Communications. 11:4743. doi: 10.1038/s41467-020-18529-y.

533 Groussin M, Gouy M. 2011. Adaptation to Environmental Temperature Is a Major Determinant

534 of Molecular Evolutionary Rates in Archaea. Molecular Biology and Evolution. 28:2661-2674.

535 doi: 10.1093/molbev/msr098.

536 Hehemann JH et al. 2010. Transfer of carbohydrate-active enzymes from marine bacteria to

537 Japanese gut microbiota. Nature. 464:908-912. doi: 10.1038/nature08937.

538 Herron MD, Doebeli M. 2013. Parallel Evolutionary Dynamics of Adaptive Diversification in

539 Escherichia coli. PLoS Biology. 11. doi: 10.1371/journal.pbio.1001490.

540 Huber CD, Kim BY, Lohmueller KE. 2020. Population genetic models of GERP scores suggest

541 pervasive turnover of constrained sites across mammalian evolution. PLOS Genetics.

542 16:e1008827. doi: 10.1371/journal.pgen.1008827.

543 Jakobsson HE et al. 2010. Short-Term Antibiotic Treatment Has Differing Long-Term Impacts

544 on the Human Throat and Gut Microbiome. PLOS ONE. 5:e9836. doi:

$545 \quad$ 10.1371/journal.pone.0009836. 
546 Ji BW, Sheth RU, Dixit PD, Tchourine K, Vitkup D. 2020. Macroecological dynamics of gut

547 microbiota. Nature Microbiology. 5:768-775. doi: 10.1038/s41564-020-0685-1.

548 Johnson ZI, Chisholm SW. 2004. Properties of overlapping genes are conserved across microbial

549 genomes. Genome Res. 14:2268-2272. doi: 10.1101/gr.2433104.

550 Karcher $\mathrm{N}$ et al. 2020. Analysis of 1321 Eubacterium rectale genomes from metagenomes

551 uncovers complex phylogeographic population structure and subspecies functional adaptations.

552 Genome Biology. 21:138. doi: 10.1186/s13059-020-02042-y.

553 Karst SM et al. 2021. High-accuracy long-read amplicon sequences using unique molecular

554 identifiers with Nanopore or PacBio sequencing. Nature Methods. 18:165-169. doi:

$555 \quad 10.1038 / \mathrm{s} 41592-020-01041-\mathrm{y}$.

556 Kenny DJ et al. 2020. Cholesterol Metabolism by Uncultured Human Gut Bacteria Influences

557 Host Cholesterol Level. Cell Host and Microbe. 28:245-257.e6. doi:

$558 \quad 10.1016 /$ j.chom.2020.05.013.

559 Kimura M. 1983. The Neutral Theory of Molecular Evolution. 1st ed. Cambridge University

$560 \quad$ Press doi: $10.1017 / \mathrm{CBO} 9780511623486$.

561 Korem T et al. 2015. Growth dynamics of gut microbiota in health and disease inferred from

562 single metagenomic samples. Science. 349:1101-1106. doi: 10.1126/science.aac4812.

563 Lawrie DS, Petrov DA. 2014. Comparative population genomics: power and principles for the

564 inference of functionality. Trends in Genetics. 30:133-139. doi: 10.1016/j.tig.2014.02.002. 
565 Leffler EM et al. 2012. Revisiting an Old Riddle: What Determines Genetic Diversity Levels

566 within Species? PLOS Biology. 10:e1001388. doi: 10.1371/journal.pbio.1001388.

567 Levins R, Lewontin RC. 1987. The dialectical biologist. Harvard University Press: Cambridge, 568 Mass.

569 Levy SF et al. 2015. Quantitative evolutionary dynamics using high-resolution lineage tracking.

570 Nature. 519:181-186. doi: 10.1038/nature14279.

571 Ley RE et al. 2005. Obesity alters gut microbial ecology. Proceedings of the National Academy

572 of Sciences of the United States of America. 102:11070-11075. doi: 10.1073/pnas.0504978102.

573 Ley RE, Turnbaugh PJ, Klein S, Gordon JI. 2006. Microbial ecology: human gut microbes

574 associated with obesity. Nature. 444:1022-1023. doi: 10.1038/4441022a.

575 Lieberman TD et al. 2014. Genetic variation of a bacterial pathogen within individuals with

576 cystic fibrosis provides a record of selective pressures. Nature Genetics. 46:82-87. doi:

$577 \quad 10.1038 /$ ng.2848.

578 Lieberman TD. 2018. Seven Billion Microcosms: Evolution within Human Microbiomes.

579 mSystems. 3:e00171-17, /msystems/3/2/msys.00171-17.atom. doi: 10.1128/mSystems.00171-17.

580 Lilja EE, Johnson DR. 2019. Substrate cross-feeding affects the speed and trajectory of

581 molecular evolution within a synthetic microbial assemblage. BMC Evolutionary Biology.

582 19:129. doi: 10.1186/s12862-019-1458-4.

583 Lin M, Kussell E. 2017. Correlated Mutations and Homologous Recombination Within Bacterial 584 Populations. Genetics. 205:891-917. doi: 10.1534/genetics.116.189621. 
585 Lin M, Kussell E. 2019. Inferring bacterial recombination rates from large-scale sequencing

586 datasets. Nat Methods. 16:199-204. doi: 10.1038/s41592-018-0293-7.

587 Lindblad-Toh K et al. 2011. A high-resolution map of human evolutionary constraint using 29

588 mammals. Nature. 478:476-482. doi: 10.1038/nature10530.

589 Locey KJ, Lennon JT. 2019. A Residence Time Theory for Biodiversity. The American

590 Naturalist. 194:59-72. doi: 10.1086/703456.

591 Lou YC et al. 2021. Infant gut strain persistence is associated with maternal origin, phylogeny,

592 and functional potential including surface adhesion and iron acquisition. bioRxiv.

$593 \quad 2021.01 .26 .428340$. doi: 10.1101/2021.01.26.428340.

594 Ludington WB, Ja WW. 2020. Drosophila as a model for the gut microbiome. PLOS Pathogens.

595 16:e1008398. doi: 10.1371/journal.ppat.1008398.

596 Lynch M et al. 2014. Evolutionary cell biology: Two origins, one objective. PNAS. 111:16990-

597 16994. doi: 10.1073/pnas.1415861111.

598 Lynch M. 2007. The origins of genome architecture. Sinauer Associates: Sunderland, Mass.

599 Lynch M, Marinov GK. 2015. The bioenergetic costs of a gene. Proc Natl Acad Sci USA.

600 112:15690-15695. doi: 10.1073/pnas.1514974112.

601 Lynch M, Trickovic B. 2020. A Theoretical Framework for Evolutionary Cell Biology. Journal

602 of Molecular Biology. 432:1861-1879. doi: 10.1016/j.jmb.2020.02.006.

603 Madi AN, Vos M, Murall CL, Legendre P. 2020. Title : Does diversity beget diversity in 604 microbiomes ? bioRxiv. 1-26. 
605 Martinez-Gutierrez CA, Aylward FO. 2019. Strong Purifying Selection Is Associated with

606 Genome Streamlining in Epipelagic Marinimicrobia. Genome Biology and Evolution. 11:2887-

607 2894. doi: 10.1093/gbe/evz201.

608 Milo R, Phillips R. 2016. Cell biology by the numbers. Garland Science, Taylor \& Francis

609 Group: New York, NY.

610 Moorjani $\mathrm{P}$ et al. 2016. A genetic method for dating ancient genomes provides a direct estimate

611 of human generation interval in the last 45,000 years. PNAS. 113:5652-5657. doi:

$612 \quad 10.1073 /$ pnas.1514696113.

613 Nayfach S, Rodriguez-Mueller B, Garud N, Pollard KS. 2016. An integrated metagenomics

614 pipeline for strain profiling reveals novel patterns of transmission and global biogeography of

615 bacteria. bioRxiv. 031757. doi: 10.1101/031757.

616 Nayfach S, Shi ZJ, Seshadri R, Pollard KS, Kyrpides NC. 2019. New insights from uncultivated

617 genomes of the global human gut microbiome. Nature. 568:505-510. doi: 10.1038/s41586-019-

$618 \quad 1058-\mathrm{x}$.

619 Neher RA, Shraiman BI. 2012. Fluctuations of Fitness Distributions and the Rate of Muller's

620 Ratchet. Genetics. 191:1283-1293. doi: 10.1534/genetics.112.141325.

621 Neher RA, Shraiman BI. 2011. Statistical genetics and evolution of quantitative traits. Rev. Mod.

622 Phys. 83:1283-1300. doi: 10.1103/RevModPhys.83.1283.

623 Nguyen $\mathrm{Ba}$ AN et al. 2019. High-resolution lineage tracking reveals travelling wave of

624 adaptation in laboratory yeast. Nature. 575:494-499. doi: 10.1038/s41586-019-1749-3. 
625 Nicolaisen LE, Desai MM. 2012. Distortions in Genealogies Due to Purifying Selection.

626 Molecular Biology and Evolution. 29:3589-3600. doi: 10.1093/molbev/mss170.

627 Pasolli E et al. 2019. Extensive Unexplored Human Microbiome Diversity Revealed by Over

628 150,000 Genomes from Metagenomes Spanning Age, Geography, and Lifestyle. Cell. 176:649-

629 662.e20. doi: 10.1016/j.cell.2019.01.001.

630 Pathria RK, Beale PD. 2011. Statistical mechanics. 3rd ed. Elsevier/Academic Press:

631 Amsterdam; Boston.

632 Pearce MT, Fisher DS. 2019. Rapid adaptation in large populations with very rare sex: Scalings

633 and spontaneous oscillations. Theoretical Population Biology. 129:18-40. doi:

$634 \quad 10.1016 /$ j.tpb.2017.11.005.

635 Pollard KS, Hubisz MJ, Rosenbloom KR, Siepel A. 2010. Detection of nonneutral substitution

636 rates on mammalian phylogenies. Genome Res. 20:110-121. doi: 10.1101/gr.097857.109.

637 Powell JE, Leonard SP, Kwong WK, Engel P, Moran NA. 2016. Genome-wide screen identifies

638 host colonization determinants in a bacterial gut symbiont. Proc Natl Acad Sci U S A.

639 113:13887-13892. doi: 10.1073/pnas.1610856113.

640 Poyet $\mathrm{M}$ et al. 2019. A library of human gut bacterial isolates paired with longitudinal

641 multiomics data enables mechanistic microbiome research. Nat Med. 25:1442-1452. doi:

$642 \quad 10.1038 / \mathrm{s} 41591-019-0559-3$. 
643 Ramiro RS, Durão P, Bank C, Gordo I. 2020. Low mutational load and high mutation rate

644 variation in gut commensal bacteria. PLOS Biology. 18:e3000617. doi:

645 10.1371/journal.pbio.3000617.

646 Romiguier J et al. 2014. Comparative population genomics in animals uncovers the determinants

647 of genetic diversity. Nature. 515:261-263. doi: 10.1038/nature13685.

648 Roodgar M et al. 2020. Longitudinal linked read sequencing reveals ecological and evolutionary

649 responses of a human gut microbiome during antibiotic treatment. bioRxiv. 2019.12.21.886093.

650 doi: $10.1101 / 2019.12 .21 .886093$.

651 Rosen MJ, Davison M, Bhaya D, Fisher DS. 2015. Fine-scale diversity and extensive

652 recombination in a quasisexual bacterial population occupying a broad niche. Science.

653 348:1019-1023. doi: 10.1126/science.aaa4456.

654 Rowland I et al. 2018. Gut microbiota functions: metabolism of nutrients and other food

655 components. Eur J Nutr. 57:1-24. doi: 10.1007/s00394-017-1445-8.

656 Ruiz L, Motherway MO, Lanigan N, Sinderen D van. 2013. Transposon Mutagenesis in

657 Bifidobacterium breve: Construction and Characterization of a Tn5 Transposon Mutant Library

658 for Bifidobacterium breve UCC2003. PLOS ONE. 8:e64699. doi:

659 10.1371/journal.pone.0064699.

660 Sakoparnig T, Field C, van Nimwegen E. 2021. Whole genome phylogenies reflect the

661 distributions of recombination rates for many bacterial species Nourmohammad, A, editor. eLife.

662 10:e65366. doi: 10.7554/eLife.65366. 
663 Sberro H et al. 2019. Large-Scale Analyses of Human Microbiomes Reveal Thousands of Small,

664 Novel Genes. Cell. 178:1245-1259.e14. doi: 10.1016/j.cell.2019.07.016.

665 Scerri EML et al. 2018. Did Our Species Evolve in Subdivided Populations across Africa, and

666 Why Does It Matter? Trends in Ecology \& Evolution. 33:582-594. doi:

667 10.1016/j.tree.2018.05.005.

668 Schloissnig S et al. 2013. Genomic variation landscape of the human gut microbiome. Nature.

669 493:45-50. doi: 10.1038/nature11711.

670 Shapiro B. Jesse. 2016. How clonal are bacteria over time? Evolutionary Biology doi:

$671 \quad 10.1101 / 036780$.

672 Shapiro B Jesse. 2016. How clonal are bacteria over time? Current Opinion in Microbiology.

673 31:116-123. doi: 10.1016/j.mib.2016.03.013.

674 Shapiro BJ et al. 2012. Population genomics of early events in the ecological differentiation of

675 bacteria. Science. 335:48-51. doi: 10.1126/science.1218198.

676 Slobodkin LB. 1980. Growth and regulation of animal populations. 2d enl. ed. Dover

677 Publications: New York.

678 Smith JM, Smith NH, O’Rourke M, Spratt BG. 1993. How clonal are bacteria? Proceedings of

679 the National Academy of Sciences. 90:4384-4388. doi: 10.1073/pnas.90.10.4384.

680 Spanogiannopoulos P, Bess EN, Carmody RN, Turnbaugh PJ. 2016. The microbial pharmacists

681 within us: A metagenomic view of xenobiotic metabolism. doi: 10.1038/nrmicro.2016.17. 
682 Taylor LR. 1961. Aggregation, Variance and the Mean. Nature. 189:732-735. doi:

$68310.1038 / 189732 \mathrm{a} 0$.

684 Tenaillon $\mathrm{O}$ et al. 2016. Tempo and mode of genome evolution in a 50,000-generation

685 experiment. Nature. 536:165-170. doi: 10.1038/nature18959.

686 Tett A et al. 2019. The Prevotella copri Complex Comprises Four Distinct Clades

687 Underrepresented in Westernized Populations. Cell Host and Microbe. 26:666-679.e7. doi:

$688 \quad 10.1016 /$ j.chom.2019.08.018.

689 Thibault D et al. 2019. Droplet Tn-Seq combines microfluidics with Tn-Seq for identifying

690 complex single-cell phenotypes. Nature Communications. 10:5729. doi: 10.1038/s41467-019-

$691 \quad 13719-9$.

692 Thomas AM et al. 2019. Metagenomic analysis of colorectal cancer datasets identifies cross-

693 cohort microbial diagnostic signatures and a link with choline degradation. Nature Medicine.

694 25:667-678. doi: 10.1038/s41591-019-0405-7.

695 Tropini C, Earle KA, Huang KC, Sonnenburg JL. 2017. The Gut Microbiome: Connecting

696 Spatial Organization to Function. Cell Host and Microbe. 21:433-442. doi:

697 10.1016/j.chom.2017.03.010.

698 Truong DT, Tett A, Pasolli E, Huttenhower C, Segata N. 2017. Microbial strain-level population

699 structure and genetic diversity from metagenomes. Genome Res. 27:626-638. doi:

$700 \quad 10.1101 / \mathrm{gr} .216242 .116$ 
701 Vallianou NG, Stratigou T, Tsagarakis S. 2018. Microbiome and diabetes: Where are we now?

702 Diabetes Res Clin Pract. 146:111-118. doi: 10.1016/j.diabres.2018.10.008.

703 Vos M, Didelot X. 2009. A comparison of homologous recombination rates in bacteria and

704 archaea. ISME Journal. 3:199-208. doi: 10.1038/ismej.2008.93.

705 Xue KS et al. 2017. Parallel evolution of influenza across multiple spatiotemporal scales Neher,

706 RA, editor. eLife. 6:e26875. doi: 10.7554/eLife.26875.

707 Yaffe E, Relman DA. 2020. Tracking microbial evolution in the human gut using Hi-C reveals

708 extensive horizontal gene transfer, persistence and adaptation. Nat Microbiol. 5:343-353. doi:

$709 \quad 10.1038 / \mathrm{s} 41564-019-0625-0$.

710 Yano JM et al. 2015. Indigenous Bacteria from the Gut Microbiota Regulate Host Serotonin

711 Biosynthesis. Cell. 161:264-276. doi: 10.1016/j.cell.2015.02.047.

712 Zhao S et al. 2019. Adaptive Evolution within Gut Microbiomes of Healthy People. Cell Host \&

713 Microbe. 25:656-667.e8. doi: 10.1016/j.chom.2019.03.007.

714 Zheng W et al. 2020. Microbe-seq: high-throughput, single-microbe genomics with strain

715 resolution, applied to a human gut microbiome. bioRxiv. 2020.12.14.422699. doi:

$716 \quad 10.1101 / 2020.12 .14 .422699$.

717 Zhu B, Wang X, Li L. 2010. Human gut microbiome: The second genome of human body. doi:

$718 \quad 10.1007 / \mathrm{s} 13238-010-0093-\mathrm{z}$.

719 Zhu Q et al. 2019. Phylogenomics of 10,575 genomes reveals evolutionary proximity between

720 domains Bacteria and Archaea. Nat Commun. 10:5477. doi: 10.1038/s41467-019-13443-4. 
721 Zimmermann M, Zimmermann-Kogadeeva M, Wegmann R, Goodman AL. 2019. Separating

722 host and microbiome contributions to drug pharmacokinetics and toxicity. Science. 363. doi:

$72310.1126 /$ science.aat9931.

724 Zlitni S et al. 2020. Strain-resolved microbiome sequencing reveals mobile elements that drive

725 bacterial competition on a clinical timescale. Genome Medicine. 12:50. doi: 10.1186/s13073-

$726 \quad 020-00747-0$.

727

728

729

730

731

732

733

734

735

736

737

738

739

740

741

742 


\section{Figures}

744 Fig. 1: a) The relationship between synonymous divergence on the $\mathrm{x}$-axis $\left(d_{S}\right)$ and the ratio of

745 nonsynonymous and synonymous divergences $\left(d_{N} / d_{S}\right)$ on the y-axis follows the form predicted

746 by purifying selection across species (Eq. S8 from Garud, Good et al. (2019)). Though by color-

747 coding individual species, we see that data points tend to be grouped by species identity, where

748 certain species fall above or below the prediction. b) By grouping genes by their pathways and

749 generating an appropriate null distribution via permutation, we can identify pathways that, under

750 the assumptions of the model, are under stronger or weaker purifying selection than expected by

751 chance. We can then examine how the mean $d_{N} / d_{S}\left(\left\langle d_{N} / d_{S}\right\rangle\right)$ of a given pathway relates to its

752 variance $\left(\sigma_{d_{N} / d_{S}}^{2}\right)$,), where the variance increases slightly faster than the square of $\left\langle d_{N} / d_{S}\right\rangle$,

753 suggesting that the coefficient of variation is greater than one (inset figure in b). c) By inverting

754 our permutation scheme, we can identify the set of species that are subject to stronger or weaker

755 purifying selection than expected by chance.

756

757

758

759

760

761 
bioRxiv preprint doi: https://doi.org/10.1101/2021.03.02.433642; this version posted March 3, 2021. The copyright holder for this preprint (which was not certified by peer review) is the author/funder, who has granted bioRxiv a license to display the preprint in perpetuity. It is made available under aCC-BY 4.0 International license.

762

a

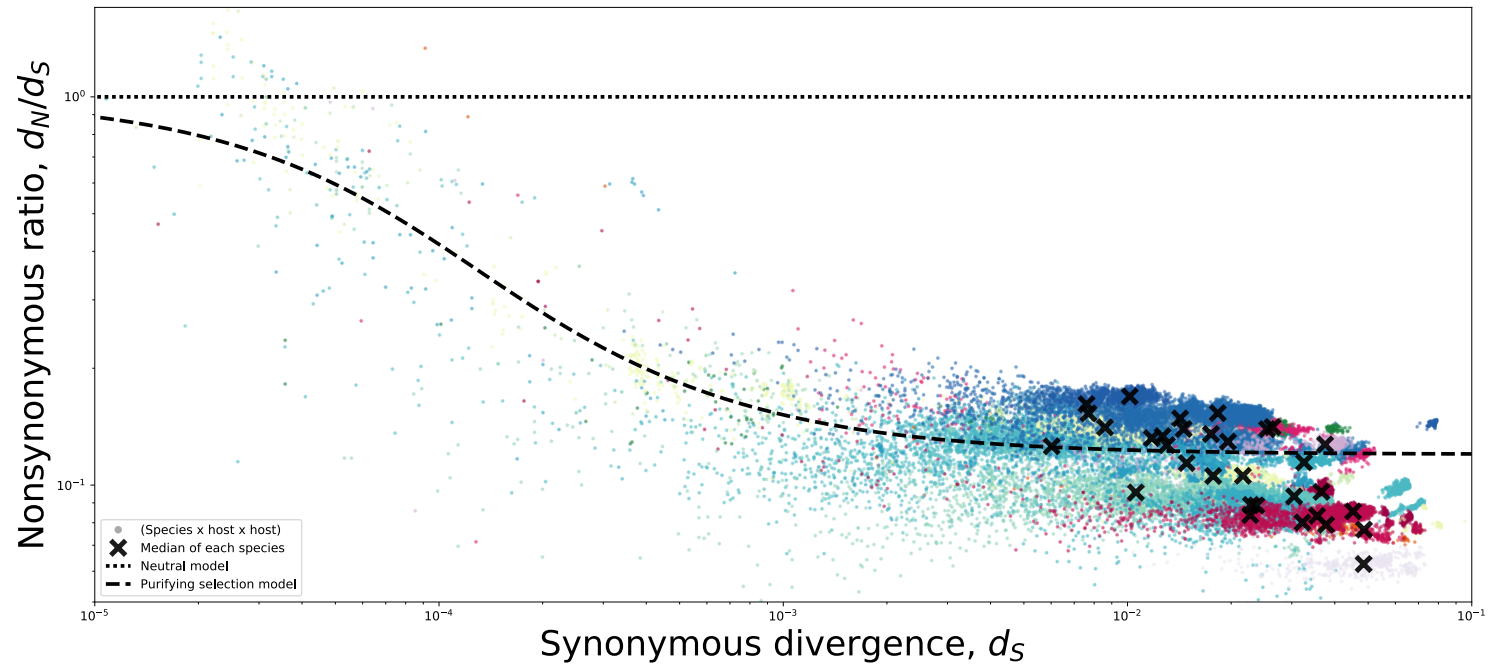

b

c
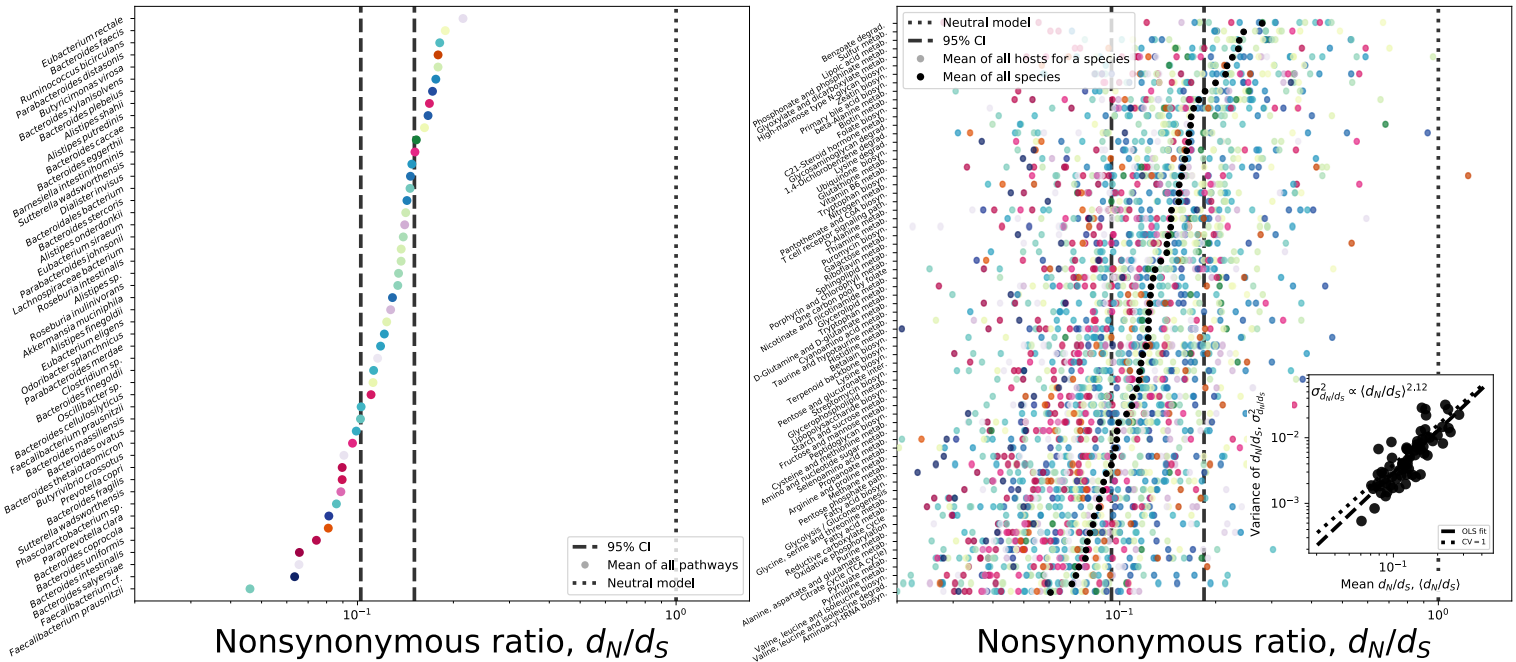